\title{
Assessing Learners' Comprehension of Logical Connectives in L2 Texts
}

\author{
Zorana Vasiljevic \\ Faculty of Literature, Bunkyo University, Koshigaya, Japan
}

\begin{abstract}
To successfully comprehend a text, readers must be able to establish coherent representation of its meaning. Construction of coherent text representation presupposes an ability to identify coherence relations that bind discourse segments together. These relations can be implicit or marked by a variety of linguistic devices such as logical connectives and signaling phrases. While there has been a growing awareness among the teachers and EFL material writers about the important role that knowledge of marker words plays in comprehension of $\mathrm{L} 2$ discourse, there is only a limited number of suitable test designs that allow assessment of learners' understanding of these words. The current paper summarizes the major findings of the research on the role of discourse markers in text processing and presents two test formats that could be used to measure students' understanding of conjunctions as text structure markers: a 'paraphrase' judgment task and text diagrams. The advantages and potential limitations of each test format are examined from both the teacher and the student perspectives.
\end{abstract}

Index Terms - logical connectives, reading comprehension, testing, sentence judgment task, text diagrams

\section{INTRODUCTION}

Comprehension entails development of coherent cognitive representations of semantic relationships in the texts. Mental representations of text meaning are built through interaction of various types of knowledge that the reader has about the world, text structure and the language itself. The construction of mental representations during reading requires from the reader ability to relate and integrate information from different segments of the text (van Dijk \& Kintsch, 1983). Relations that hold together different segments of the discourse are referred to as coherence relations. They can be implicit or explicit. While marking of coherence relations is not restricted to discourse markers, they are by far the most frequently studied discourse signaling tools.

Discourse markers include a large number of linguistic devices, some of which are grammatical and some of which are lexical in nature. Some discourse markers are used mostly in spoken language, while others are more common in formal writing styles. What they all have in common is that while their specific meaning is determined by the context, their core meaning is procedural, rather than conceptual (Fraser, 1999). They serve as directional guides for text receivers about how the incoming information should be interpreted and integrated with the preceding discourse segments (Halliday \& Hasan, 1976; Carpenter \& Just, 1977), show the speaker's attitude to the content of the discourse (Swan, 1980), and indicate the relative importance of the ideas in the text (Jung, 2003). This means that while coherence relation are an intrinsic part of cognitive representations, linguistic markers are a surface code that can facilitate the process of formation of coherent text representations (Sanders \& Noordman, 2000).

Based on their signaling function, discourse markers can be broadly divided into four groups: 1) previews (e.g., There are four stages...); 2) summarizers (e.g., To sum up so far...); 3) emphasis markers (e.g., This is the key), and 4) logical connectives (e.g., and, or, first, second...) (Jung, 2003). Logical connectives, also referred to as conjunctions, contribute to text cohesion by indicating the way in which a text segment is connected to what comes before or after. They serve as cues for the reader about how the text should be interpreted and contribute to the overall coherence of the discourse.

Halliday and Hasan (1976) identify four main types of conjunctive relations: 1) additive (e.g. and, in addition, furthermore....etc.); 2) adversative (e.g. but, yet, on the contrary...etc.); 3) causal (e.g. so, therefore...etc.); 4) temporal (e.g. then, next, subsequently...etc.). Another common type is illustrative conjunctions, which signal exemplification or further amplification of some aspect of the preceding information unit (Pretorius, 2006).

While it seems plausible to expect that explicit signaling of coherence relations would have a positive effect on text comprehension and the subsequent information recall, the existing empirical work has produced mixed results. Explicit discourse markers were found to improve performance of less-skilled readers in L1 (Marshall \& Glock, 1978-1979; Meyer, Brandt \& Bluth, 1981; Geva \& Ryan, 1985), and to enhance comprehension of academic lectures of second language learners (Flowerdew, \& Tauroza, 1995; Jung, 2003, 2006). There is also some evidence to suggest that text signaling results in the employment of more effective reading strategies. Loman and Mayer (1983) reported that signal words encouraged students to use more meaningful reading strategies as opposed to rote reading. Without the discourse cues, readers often perceive a text as a list of separate arbitrary units that need to be memorized. Conjunctions serve as text-structure markers, directing the reader's attention to the compositional aspects of the passage and helping them to 
organize conceptual idea units into a coherent whole. Knowledge of logical connectives was also found to facilitate comprehension of the texts from unfamiliar domains. This is because, without the sufficient content schemata, readers tend to resort to the surface aspects of the texts and linguistic devices to help them to make inferences about the relationships between propositions in the text (Goldman \& Murray, 1992). Experimental data also indicate that explicit marking of coherence relations leads to faster text processing (Haberlandt, 1982; Millis \& Just, 1994; Sanders \& Norman, 2000) and better recall of sentence-pairs (Caron, Micko \& Thuring, 1988).

There are, however, some studies in which signaling cues were found no have no effect or only a limited impact on text comprehension. Firstly, some studies (e.g., Taboada, 2006) suggest that as much as $60 \%$ to $70 \%$ of semantic relations in both spoken and written corpora may be unmarked. Secondly, even when coherence relations are signaled in the text, it is not clear to what extent language receivers are aware of their presence (Sanders, Spooren \& Noordman, 1993; Millis, Graesser \& Haberlandt, 1993). In some studies, inclusion or elimination of conjunctions was found to have little effect on the perceived difficulty of the text. For example, in a series of experiments that Millis (1993) and her colleagues conducted, the reading difficulty ratings and reading times were equivalent for the no-connective and connective versions of the texts. Thirdly, the effect of discourse markers seems to depend on the nature of the relationship they mark and their position in the text. Chaudron and Richards (1986) found that while presence of macromarkers (i.e., discourse signals that indicate main transition points in the texts) improves listening comprehension of second language learners, micromarkers (i.e., cues that indicate inter-sentential relations) had no effect on student performance. Similar results were obtained by Chung (2000) who found that the presence of discourse markers facilitated understanding at the macrostructure level of reading, but had no effect at microstructure level between the segments of reading texts. Al-Surmi (2011) examined whether the presence of discourse markers improves text comprehension at a global level and found that explicit signaling of coherence relations had no effect on student performance. Finally, data from experimental studies suggests that inclusion of discourse markers does not necessarily facilitate retention of information. Millis et al. (1993) investigated the effect that the presence of discourse markers had on memory for expository texts and found that, not only did they not enhance the reader's memory, but on the contrary, they seemed to lead to fragmentation of cognitive representation of text information and interfere with its subsequent recall. Sanders and Noordman (2000) observed that, although explicit signaling phrases can increase the speed of text processing, this advantage seems to be limited to the encoding of coherence relations. Linguistic markers were found to have little effect on the subsequent recall of the propositions in the text.

The conflicting results of experimental research can be attributed to several factors. Firstly, there seem to be significant differences in distribution of discourse markers in spoken and written discourse. The study by Louwerse and Mitchell (2003) revealed as much as a 10 times higher incidence of the occurrence of discourse markers in spoken language. Marker words were also found to be twice as common in informal than in formal discourse. The discrepancies in the findings may also have resulted from the differences in distribution and function of marker words in different academic disciplines (Geva, 1992). Readers in different disciplines encounter different types of conjunctions, which often have diverse functions within a text, and which differ in the extent to which they contribute to text cohesion or facilitate the processing of the text. The failure to obtain the effect of signaling may also be due to the fact that many studies adopted the number of recalled information units as the standard of comparison for the student performance on signaled and non-signaled passages. As Loman and Mayer (1983) observed, the positive effect of signal words may not be reflected in the number of ideas recalled, but rather in their quality. While readers in both conditions may recall the same number of ideas, non-signaled versions often result in readers focusing on the first and last few ideas in the passage (primacy and recency effect), while signaled texts direct the reader's attention to the key conceptual information in the text. Another reason for the discrepancies in the findings may lie in differences in the processing load for different types of logical connectors. Some types of connectives are intrinsically more complex than the others. For example, while temporal connectives denote only time-order, causal connectives encompass both temporal and causal features (Millis et al., 1993). Furthermore, the processing load that connectives impose on the reader will also depend on whether the direction of perception (i.e., the direction of eye movement along text) coincides or collides with the direction or reasoning within the reader's mind (Ozono \& Ito, 2003). Connectives that mark continuity in a discourse (i.e., additive, temporal, illustrative and causal relations) are processed more easily than adversative relations that signal discontinuity (Vonk \& Noordman, 1990; Murray, 1997; Pretorius, 2006). As a result, without explicit marking adversative relationships are considerably more difficult to infer than temporal or causal relationships (Millis et al., 1993). The effect that connectives have on comprehension also depends on the extent to which the semantic relation that they mark contributes to the coherence of the text. For example, Sanders and Noordamn (2000) found that problem-solution relation contributes to text organization more than a listing relation. The results of the studies may also have been affected by manipulation of natural distribution of linguistic markers. In some studies, conjunctions were intentionally added to texts, which may have had a negative effect on the recall of propositions. As Millis (1993) and her colleagues observed, connectives that are unnatural to the normal interpretation of the text may have a negative effect on memory. Another factor to consider is the learners' proficiency level. Learners at lower levels of proficiency were found to be more affected by the type of logical relations and explicitness of discourse markers than higher-level learners (Nippold, Schwartz \& Undlin, 1992; Chung, 2000; Ozono \& Ito 2003). Finally, the differences in the results may have also been caused by the factors such as learners' familiarity with the formal and content schema, text type 
(e.g., narrative vs. expository texts), and assessment procedures (Jung, 2003) and general frequency of occurrence of different logical connectives in natural language (Nippold et al., 1992).

While contradictory finings raise questions about the extent to which explicitness of linguistic markers can be expected to facilitate post-reading recall of the propositions in the text, there seems to be sufficient experimental support for the positive effect that logical connectors have on real-time text processing and construction of meaning. Several conditions, however, must be fulfilled for this effect to take place. According to Goldman and Murray (1992), in order to take advantage of the linguistic markers in the text, readers must lack the requisite linguistic knowledge and content schema to infer implicit coherence relations. High-skilled readers with sufficient background knowledge, good understanding of the text structure, and a high level of language proficiency are able to construct coherent mental representations of the texts, even when logical relationships are not explicitly signaled. Secondly, readers must be familiar with the general functions of discourse markers. Thirdly, they must be able to instantiate those functions in the specific text in which the signal words occur. In addition to these conditions, Jung (2003) also observed that discourse cues are more likely to facilitate comprehension of expository texts than narrative texts, as they tend to incorporate more complex semantic relationships that go beyond the simple episodic sequences.

Based on the outline of the conditions above, it is possible to assume that the presence of discourse markers will be beneficial to second language learners, who often lack the necessary cultural schemata and whose limited vocabulary size may not allow them to take full advantage of the lexical cohesion in the text. Logical connectors provide explicit information about the relation between text segments, and therefore they can serve as an additional orientating tool for readers about how to interpret the text message, reducing the probability of misinterpretation of the text message and communication breakdown. Effective use of text-structure markers is also expected to lead to better allocation of learners' mental resources. Without a conjunction, the reader must rely on the context and world knowledge to a construct a coherent link between the segments of the text, which requires more mental elaboration and places additional pressure on the working memory. The presence of conjunctions reduces the need for inference, and consequently reduces the likelihood of cognitive overload during the processing of L2 texts.

Learners at the intermediate level, in particular, are likely to benefit from the presence of signal words in the texts. Low-level learners, like poor readers in L1, tend to focus on smaller text units and, therefore, may fail to take advantage of the presence of connecting signals, while high-level learners are likely to be able to infer coherence relations and integrate text units, even when signal words are not explicitly stated (Kintsch, 1990; Geva, 1992). Furthermore, a higher level of complexity of semantic relations in expository texts means that knowledge of logical connectives will be particularly important for learners who need English for academic purposes. At the tertiary level, students are expected to read massive amounts of print information, understand the texts accurately and synthesize information from various sources into coherent knowledge bases on content-related topics (Pretorius, 2006). Therefore, the ability to comprehend logical relations in expository texts can have a significant effect on student academic performance. As early as 1978, Cohen and Fine observed that non-native adult speakers of English frequently overlook cohesive textual links and, as a result, have difficulties in comprehending expository texts. In studies conducted by Olsen and Huckin (1990) and Clerehan (1995), inability to utilize discourse cues was reported as one of the main reasons why students were not able to distinguish between the main ideas and the supporting details in academic lectures. Pretorius (2006) found that difficulties in understanding causal and adversative relations in expository texts can significantly undermine a student's academic performance.

These findings clearly suggest the need for activities that would help learners to understand logical connectives and make them attend more carefully to the semantic relations that they mark. In order to be able to help learners to take advantage of signal words in the texts, however, it is important that teachers can accurately measure the level of student understanding of the functions that these words perform in the texts. Some popular test formats include activities where learners are asked to identify conjunctions in the text that indicate a specific relationship, multiple-choice activities where students have to select the linking word that fits best the particular sentence context, and "fill-in the-blanks" type activities where learners need to insert linking words in a passage. While these types of activities can be used for teaching purposes, they are not so well-suited when it comes to testing learner comprehension of logical connectors in L2 texts. Learners may be able to identify marker words for a particular semantic relationship without really understanding the text itself. Multiple-choice activities require learners to pay attention to surrounding context, but the options often consist of conjunctions from different semantic categories, which means that even with only a very general understanding of the context, learners may be able to select the correct answers. The fill-in-the gap activity forces learners to take the whole text into account, but for the correct completion of the task learners need to have productive knowledge of transition words. The purpose of this paper, therefore, is to examine some alternative activities that can be used to assess learner understanding of logical connectors as used in the texts. Two test formats will be introduced: a 'paraphrase' judgment task and text diagrams. A sample of each test will be provided and the advantages and potential limitations of each format will be discussed.

\section{TEST I (PARAPHRASE JUdGMENT TASK)}

In a traditional paraphrasing task, students are given a sentence in L2 and asked to write a sentence equivalent in meaning. A part of the paraphrase may be given to restrict students to a particular grammatical structure that is being 
tested. To write a good paraphrases, students must have a good understanding of the original sentence, and consequently, their responses on a paraphrasing task can indicate difficulties that they may be experiencing with comprehension. For testing purposes, however, a potential problem with the traditional paraphrasing task is that it requires productive language abilities. When the purpose of the test is assessment of comprehension, it is important that student performance is not affected negatively by their poor command of sentence structure or limited vocabulary knowledge; otherwise the validity of the test itself may be undermined. Therefore, for the purpose of assessing student comprehension of logical connectives in a particular text, a traditional paraphrasing task has been modified into a same-meaning sentence decision task, where the students are provided with a sentence from the original text and its "paraphrase" and asked to judge whether the two sentences have the same or different meaning. (For a sample of this task, see Appendices A and B.)

The 'paraphrasing' judgment task as a test format offers several potential advantages. Firstly, by asking students to judge whether two sentences have the same or different meanings, instructors ensure that the test is truly measuring text comprehension.

Secondly, the judgment task offers instructors the flexibility to manipulate the specific variables upon which they want students to be assessed. As the purpose of the test is to demonstrate comprehension of logical connectors, vocabulary can be replaced with simple synonyms or even left unchanged, ensuring that unfamiliar words and expressions do not interfere with the student's decision-making processes.

The paraphrasing judgment task enables teachers to draw learner attention to the specific logical connectives. As discussed before, logical relationships differ in the amount of processing load that they impose on the readers. The paraphrasing judgment task allows teachers to approach logical connectives individually and focus on those relationships that students have most difficulty with, or that are particularly important for constructing the meaning in the types of texts that students are expected to read. For example, for learners who need English for academic purposes and who are expected to read large amounts of expository texts, the focus should be on the causal and adversative relations, as they tend to be frequent in this text type, as well as pose difficulties for the students.

Another advantage of the paraphrasing judgment task is that the target sentences can be either text-based or independent. This means that with this test format instructors can assess both the level of student understanding of semantic relations in particular texts, as well as their general understanding of different types of logical relations. Furthermore, the sentences that students need to judge can be independent of each other, so that each test item represents "a fresh start" for the test-taker. This makes the test results more reliable and enables teachers to get a better picture of student understanding of particular discourse markers.

Another advantage of this form of this test format is that it is quick and easy to mark. A judgment task is basically a form of true/false test, which offers rapid and reliable scoring.

Finally, the test is likely to have a positive backwash effect on learners. Regular encounters with this type of test should make learners pay more attention to the discourse markers in L2 texts that they encounter in class, which should, consequently, help them to develop more coherent and meaningful interpretations of the texts.

Like other forms of assessment, a paraphrasing judgment task is not without its limitations. One possible downside of this test format is that the test scores do not provide much information about the student overall level of text comprehension. This effect, however, can be counteracted by increasing the number of test items. Another potential problem is that some students may try to guess the answers, and with only two options, they have a $50 \%$ chance of choosing the correct answer by chance alone. Experimental studies (e.g. Ebel, 1979; Downing, 2003), however, have shown that random guessing on its own is highly unlikely to result in a high test-score. Furthermore, true-false tests were found to be neither more nor less reliable than widely used multiple-choice tests (Tasdemir, 2010) and significantly more reliable than essay-based examination (Gates, 1921). According, to Gates (1921) combining several true-false tests can produce markedly higher correlation with the criterion than essay writing, which in turn increases the predictive value of the test. The probability of guessing can also be reduced by asking learners to provide reasons behind their decisions. Finally, it should be remembered that a paraphrasing judgment task is not intended to be a measure of a student's overall reading ability or their language proficiency. What it does is measure student knowledge of the specific sub-skill - comprehension of discourse markers in the text - and it can be used to check on a student's progress with it. For more formal assessment purposes, this test format can be integrated with other common testing techniques.

\section{TEST II (TEXT DiAgRAM)}

Another way in which students can be helped to recognize structural patterns in texts is through the use of graphic organizers. A graphic organizer can be defined as "a visual and graphic display that depicts the relationships between facts, terms, and ideas within a learning task" (Hall \& Strangman, 2002, p. 1). Some common forms of graphic organizers include semantic maps, tree diagrams, flowcharts and so on.

The results of a number of experimental studies (e.g., Cheng, Lowe \& Scaife, 2001; Vekiri, 2002; Verdi \& Kulhavy, 2002; McCrudden, Schraw, Lehma \& Poliqun, 2007) suggest that a graphic display of information in the text can improve reading comprehension. This is believed to be because graphic organizers help readers visualize not only individual elements of information, but also the relationships between them, reducing the amount of cognitive effort that 
the reader needs to invest to construct inferences necessary to connect information across the different segments of the text (McCrudden et al., 2007). The results of some studies also suggest that the application of visual displays may have a positive influence on students' attitudes towards L2 reading. Mede (2010), for example, found that instruction of graphic organizers improved students' reading performance, which helped them to develop more positive feelings about reading in English.

Although graphic organizers have mostly been used for instructional purposes, they could also prove useful in testing learner understanding of the logical-structural information that conjunctions provide in the text. In this test format, the key concepts in the text are recorded as diagram nodes and the learners are asked to add the symbols that indicate the relationships between them. A sample of this test format is available in Appendix C. (The test is based on the text provided in Appendix A.)

For text diagrams to be used for testing, it is necessary to ensure that the students are familiar with common symbols for encoding the logical relationships between the ideas in the text. A summary of the major logical relations and the corresponding symbols is provided below.

1. Cause \& Effect Symbol: $\rightarrow$

e.g. I was too tired, so I decided not to go to the party.

tired $\rightarrow$ decision not to go to the party

cause effect

2. Contrast Symbol: v.s.

e.g. I was going to go to the sports club, but I was too tired.

plan to go to the $S$. C. v.s. too tired

3. Comparison Symbol: =

e.g. Like her sister, Mary can swim very well.

swimming: Mary $=$ her sister

4. Exemplification Symbol: e.g.

e.g. There are many wonderful ski resorts in Hokkaido; for example Kiroro.

wonderful ski resorts in Hokkaido e.g. Kiroro

5. Addition Symbol: +

e.g. He is very good at math. He is also an excellent tennis player.

math $\boldsymbol{V}+$ tennis $\boldsymbol{\vee}$

6. Chronology Symbol: >>

First I went to the gym. Then I had breakfast.

went to the gym > breakfast

7. Listing Symbol: 1)...2).....3)...

There are three things you need to do to deal with culture shock when traveling abroad. First, you need to learn as much as you can about the new culture before traveling abroad. Second, you need to be patient. Finally, you need to be optimistic.

for culture shock $\downarrow:$ 1. learn bout the new culture

\section{2. be patient \\ 3. be optimistic}

Although this test format requires prior training, it is believed that introduction of text diagrams into class practices will benefit students in several ways. Visual representation of text structure is expected to increase student ability to recognize content units in the discourse. The test format encourages students to look beyond the sentence level and to attend to global coherence based on larger discourse chunks. Regular practice with this type of test could also potentially improve student note-taking skills. This means that text diagrams can become an important learning tool.

The main advantages of the text-diagram format, however, are in the testing opportunities it offers. Firstly, diagrams allow teachers to test student comprehension of the whole text. A successful completion of a text diagram requires a good understanding of both the content and the text structure. Diagrams allow assessment of learner comprehension of discourse markers at both local and global levels, and they clearly indicate the sections of the text that the learners may be having problems with. This means that text diagrams can be a very useful diagnostic tool.

Secondly, the text-diagram format properties make it potentially superior to other forms of assessment in terms of both test validity and reliability. The use of text diagrams for testing learner comprehension ensures that students' scores are not affected by their ability to express themselves in the target language, which increases the validity of the test measure. At the same time, the fact that a range of acceptable answers is restricted by the context makes the scoring process easier and more objective, and consequently the test itself more reliable.

Finally, the test format is economical in terms of the time needed for its preparation and marking. Unlike with multiple-choice tests, for which writing of good distractors requires a lot of trialing and detailed statistical analysis, or integrative tests, such as composition writing which are easy to construct but difficult and time-consuming to mark, text diagrams can be prepared relatively quickly. All teachers need to do is identify idea units and propositions in the text and transfer them into the diagram nodes, leaving it to the students to add the symbols that depict the relations among them. The close-ended nature of the task means that scoring will be rapid and straightforward. 
In addition to the benefits that the text-diagram format brings in terms of instruction and assessment, it could also prove to be a useful research tool. Diagram frames can be designed to display both implicit and explicit coherence relations in the text, and the reader's ability to complete them may provide a valuable insight into the role that discourse markers play in the text coherence and comprehension.

In short, the analysis of the text-diagram testing format suggest that this type of testing may bring multiple advantages in terms of assessment, instruction and further the understanding of the role that discourse markers play at the structural levels of the text.

\section{CONCLUSION}

The paper has summarized the major findings of the research on the role of discourse markers in text comprehension and content recall, and introduced two test formats that could be used for assessment of student levels of understanding of the use of conjunctions in L2 texts: a 'paraphrase' judgment task and a text diagram. These test formats are believed to have several potential advantages over the other commonly used assessment models, such as multiple choice and cloze tests.

First, the two test formats have several advantages in terms of test design. To begin with, both tests are criterion-referenced tests that directly and accurately measure the abilities they are intended to measure - learners' comprehension of logical connectives in L2 texts. The tests do not require any productive language skills on the part of the learner. This means that learner performance will not be impeded and their scores will not be affected by their limited ability to express themselves in the target language, which increases fairness and validity ratings of the test measure.

Furthermore, the tests are relatively easy to construct, they do not take much time to complete, marking is straightforward, and the scores can be easily interpreted by both instructors and the learners.

Thirdly, the proposed test formats can be used as diagnostic tests, progress tests and achievement tests. The fact that the connectives will often determine the final interpretation of the text means that performance on the conjunction tests can serve as an indicator for the teachers about the sections of the texts that the students may be having problems with, as well as the possible sources of these difficulties. For example, the tests can highlight the types of connectors that present problems for the learners of particular language backgrounds. An error in a paraphrasing judgment task or a wrongly completed diagram can also reveal students' lack of familiarity with the particular signal word or one of its meanings. For instance, a student may be familiar with the temporal meaning of since, but fail to recognize its causal function. The restriction of the possible answers also means that the tests could be particularly useful for assessment of learner progress and final achievement in coordinated language programs.

Finally, regular testing on comprehension of logical connectors is expected to have a positive backwash effect. As Hughes (1989) points out, assessment has a strong impact on both teaching and learning, which can be either beneficial or harmful. Teachers should, therefore, develop tests that focus on the skills that they want their students to improve, so that test preparation and feedback promote learning. In other words, tests should not be seen only as an assessment tool, but also as a learning opportunity. Regular 'pop quizzes' on comprehension of discourse markers can help teachers assess learner progress and ultimately adjust instruction to match their needs. They are also likely to keep learners on their toes. With the prospect of the test coming up, students are more likely to pay attention to structural aspects of the text, which in turn should increase their sensitivity about the links between the segments of discourse, help them recognize the important role that transition words play in text coherence, and deepen their knowledge of the function of the individual marker words. The proposed test formats are also likely to be conducive to more effective methods of studying, as they require learners to really understand the text rather than to try to commit information to memory for the purpose of sheer reproduction. Finally, regular testing should help learners develop the habit of monitoring their own progress, which should promote their independence. In short, explicit focus on discourse markers with regular assessment could help students become both more strategic readers and more autonomous learners.

\section{APPEndix A SAMPLE TEXT: WHEN AND WHERE DO TORNADOES OCCUR?}

Tornadoes that hit Washington, D.C. suburbs in September 2001 illustrate a fact often stated by weather forecasters: "Tornadoes can and do happen any time of the year in just about any location." No one knows this better than National Sever Storms Laboratory Research Meteorologist Dr. Harold Brooks, who has studied the climatology of severe weather in the United States to better understand when and where tornadoes are most likely to occur. By understanding the threat posed by tornadoes in the United States, particularly the threat of strong and violent tornadoes, forecasters can more accurately predict them and communities can better prepare for them.

Through his study of tornado climatology, Brooks has revealed several important things. First, tornadoes occur most often in the central United States, commonly known as Tornado Alley. Second, the central plains have a repeatable annual tornado cycle, with the highest probability of tornado occurrence in the springtime. Finally, areas outside of tornado alley do not have a typical tornado season and experience fewer tornadoes.

The concept of Tornado Alley may be very important for the emergency management community, Brooks said.

"It is relatively easy to keep awareness up in a region where events happen frequently and where the threat is 
confined to a relatively short period of time," he said. "In addition, it is typically easier to recruit volunteer storm spotters in such an area and to maintain their enthusiasm."

Public awareness was high, for instance, during the May 3, 1999 Oklahoma City tornado. Despite damaging almost 8,000 structures, fewer than 40 direct fatalities occurred. In addition to timely and accurate warnings from the National Weather Service and live coverage from local television and radio stations, people in the path of the tornadoes knew how to respond and did it.

In contrast, heightening awareness in an area where tornadoes rarely occur or occur over a broader season of the year is much more difficult, Brooks suggests. In fact, he believes a lack of public awareness in areas of the country where the threat of a tornado on any particular day is low is one reason for many of the high death toll events that have occurred in the past 20 years. During that period of time, only two of the 22 tornadoes in the United States have caused at least eight fatalities (representing the highest 10 percent of death tolls) in Tornado Alley. Those are the Andover, Kansas tornado on April 26, 1991, which had its fatalities in a trailer park, and the May 3, 1999, Oklahoma City tornado, which was the (inflation adjusted) biggest property damage tornado in U.S. history.

Note: Abridged from "When and Where Do Tornadoes Occur? (Retrieved: June 30, 2012)

URL: http://www.nationalatlas.gov/articles/climate/a_tornadoes.html

\section{APPENDIX B TEST I: PARAPHRASE JUdGMENT TASK}

Instructions: Choose whether the following pairs of sentences have the same or different meanings.

1. a) By understanding the threat posed by tornadoes in the United States, particularly the threat of strong and violent tornadoes, forecasters can more accurately predict them and communities can better prepare for them.

b) Because forecasters and communities can now understand, predict and prepare for tornadoes better, tornadoes in the U.S., even the strong and violent ones, present less of a threat. Same Different

2. a) It is relatively easy to keep awareness up in a region where events happen frequently and where the threat is confined to a relatively short period of time. In addition, it is typically easier to recruit volunteer storm spotters in such an area and to maintain their enthusiasm.

b) People are likely to be more alert to tornadoes and more willing to join volunteer storm spotter groups if they live in the areas where tornadoes commonly occur at specific times of the year. Same Different

3. a) Despite damaging almost 8,000 structures, fewer than 40 direct fatalities occurred.

b) About 8,000 structures were damaged and consequently there were less than 40 direct deaths. Same Different

4. a) In addition to timely and accurate warnings from the National Weather Service and live coverage from local television and radio stations, people in the path of the tornadoes knew how to respond and did it.

b) Thanks to correct and well-timed information from the National Weather Service, TV and radio, people knew what to do to prepare for the tornado. Same Different

5. a) ack of public awareness in areas of the country where the threat of a tornado on any particular day is low is one reason for many of the high death toll events that have occurred in the past 20 years.

b) Many of the tornado-related deaths in the last two decades took place because tornadoes occurred in the areas where people did not expect them and were consequently less alert to the dangers they pose. Same Different

\section{APPENDIX C TEST II: TEXT DiagRAM}

Instructions: Read text "When and Where Do Tornadoes Occur" and complete the diagram below by adding the suitable symbols to indicate the relationships between the ideas.

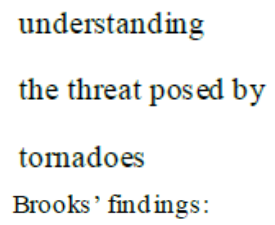

possible to predict \& prepare for

tornadoes better

1. tornadoes occur most often in the central U.S. (Tornado Alley)

2. the central plains have a repeatable annual tornado cycle (the highest frequency in the spring)

3. areas outside Tornado Alley - no typical season \& fewer tornadoes

Tornado Alley - important for the emergency management 
A. 1. easy to keep awareness

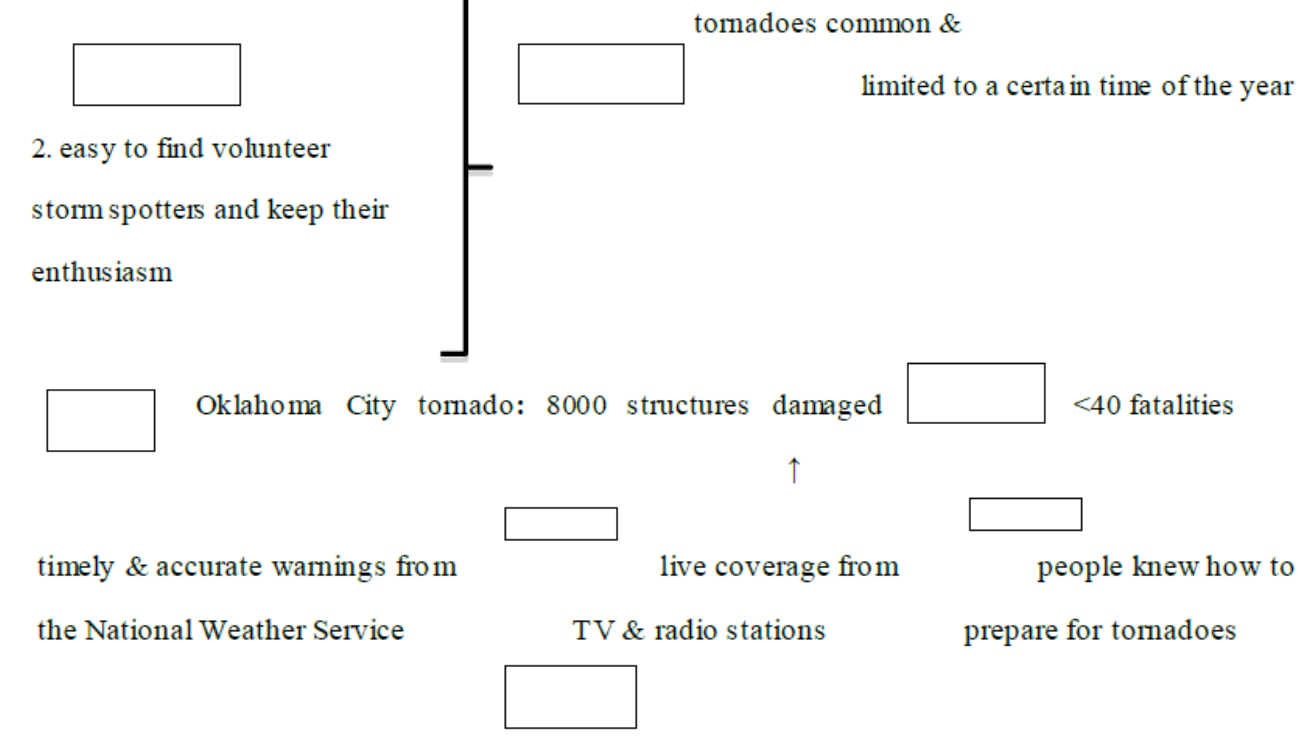

B. difficult to keep awareness

tornadoes rare / spread around the year

Brooks: lack of public awareness

the high death toll in the past 20 years

TEST II (Model Answers)

understanding

the threat posed by

possible to predict \& prepare for

tornadoes

tornadoes better

\section{(n)}

Brooks' find ings:

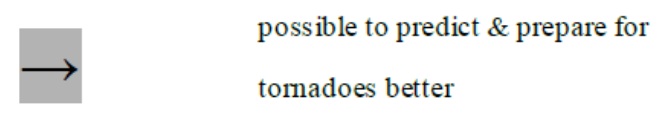

1. tornadoes occur most often in the central U.S. (Tornado Alley)

2. the central plains have a repeatable annual tomado cycle (the highest frequency in the spring)

3. areas outside Tomado Alley - no typical season \& fewer tornadoes Tomado Alley - important for the emergency management

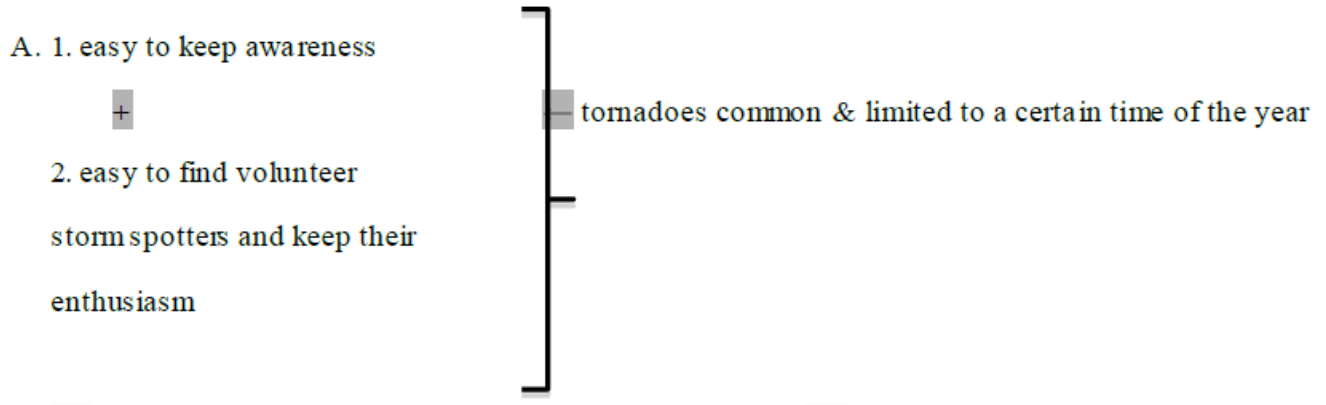

e.g. Oklaho ma City tornado: 8000 structures damaged v.s. $<40$ fatalities

$\uparrow$

timely \& accurate warnings from + live coverage + people knew how to prepare for

the National Weather Service TV\& radio stations tornadoes

V.S.

B. difficult to keep awareness $\longleftarrow$ tornadoes rare / spread around the year

Brooks: lack of public awareness $\rightarrow$ the high death toll in the past 20 years 


\section{REFERENCES}

[1] Al-Surmi, M. (2011). Discourse markers and reading comprehension: Is there an effect? Theory and Practice in Language Studies $1.12,1673-1678$.

[2] Caron, J., Micko, H.C. and Thuring, M. (1988). Conjunctions and the recall of composite sentences. Journal of Memory and Language 27, 309-323.

[3] Carpenter, P.A. \& Just, M. A. (1977). Integrative processes in comprehension. In D. LaBerge \& J. Samuals (eds.), Basic processes in reading: perception and comprehension (pp. 217-241). Hillsdale, NJ: Erlbaum.

[4] Chaudron, C. \& Richards, J. C. (1986). The effect of discourse markers on the comprehension of lectures. Applied Linguistics 7, 113-127.

[5] Cheng, P.C.H., Lowe, R.K., \& Scaife, M. (2001). Cognitive science approaches to understanding diagrammatic representations. Artificial Intelligence Review, 15.1-2, 79-94.

[6] Chung, S.L.J. (2000). Understanding signals and reading comprehension. Australian Review of Applied Linguistics 23, 77-93.

[7] Clerehan, R. (1995). Taking it down: Notetaking practices of L1 and L2 students. English for Specific Purposes 14, 135-155.

[8] Cohen, A.D. \& Fine, J. (1978). Reading history in English: Discourse analysis and the experience of native and non-native readers (Working Papers on Bilingualism No. 16). Ontario Institute for Studies in Education, Modern Language Centre, Toronto.

[9] Downing, S. M. (2003). Guessing on selected-response examinations. Medical Education 37.8, 670-671.

[10] Ebel, R.L. (1979). Essentials of educational measurement. NJ: Prentice Hall.

[11] Flowerdew, J. \& Tauroza, S. (1995). The effect of discourse markers on second language lecture comprehension. Studies in Second Language Acquisition 17, 435-478.

[12] Fraser, B. (1999). What are discourse markers? Journal of Pragmatics 31, 931-952.

[13] Gates, A. (1921). True-false test as a measure of achievement. The Journal of Educational Psychology 12.5, $276-287$.

[14] Geva, E. (1992). The role of conjunctions in L2 text comprehension. TESOL Quarterly 26, 731-747.

[15] Geva, E. and Ryan, E. B. (1985). Use of conjunctions in expository texts by skilled and less-skilled readers. Journal of Reading Behavior 17, 331-346.

[16] Goldman, S.R. \& Murray, J.D. (1992). Knowledge of Connectors as Cohesion Devices in Text: A Comparative Study of Native-English an English-as-a-Second Language Speakers. Journal of Educational Psychology 84.4, 504-519.

[17] Haberlandt, K. (1982). Reader expectations in text comprehension. In J. F. Le Ny, \& W. Kintsch, (Eds.), Language and comprehension (pp. 239-249). Amsterdam: North-Holland.

[18] Hall, T. \& Strangman, N. (2002). Graphic organizers. National Center on Assessing the General Curriculum. [Online] URL: http://www.cas.org/ncac. (accessed: 29/7/2012)

[19] Halliday, M.A.K. \& Hasan, R. (1976). Cohesion in English. UK: Longman.

[20] Hughes, A. (1989). Testing for language teachers. Cambridge: Cambridge University Press.

[21] Jung, E. H. (2003). The role of discourse signaling cues in second language listening comprehension. Modern Language Journal 87. iv, 562-577.

[22] Jung, E.H. (2006). Misunderstanding of academic monologues by nonnative speakers of English. Journal of Pragmatics 38, 1928-1942.

[23] Kintsch, E. (1990). Macroprocesses and microprocesses in the development of summarizing skill. Cognition and Instruction 7 , 161-195.

[24] Loman, N.L. and Mayer, R.E. (1983). Signaling Techniques that Increase the Understandability of Expository Prose. Journal of Educational Psychology 75.3, 402-412.

[25] Louwerse, M.M. \& Mitchell, H. (2003). Toward a taxonomy of a set of discourse markers in dialogue: a theoretical and computational linguistic account. Discourse Processes 35. 3, 243-281.

[26] Marshall, N. \& Glock, M.D. (1978-1979). Comprehension of connected discourse: A study into the relationship between the structure of text and information recalled. Reading Research Quarterly 14, 10-56.

[27] McCrudden, M.T., Schraw, G., Lehman, S. \& Poliquin, A. (2007). The effect on causal diagrams on text learning. Contemporary Educational Psychology 32, 367-388.

[28] Mede, E. (2010). The effects of instruction of graphic organizers in terms of students' attitudes towards reading in English. Procedia Social and Behavioral Sciences 2, 322-325.

[29] Meyer, B. J. F., Brandt, D.N., \& Bluth, G.J. (1981). Use of author's textual schema: Key for ninth graders' comprehension. Reading Research Quarterly 15, 72-103.

[30] Millis, K., Graesser, A. \& Haberlandt, K. (1993). The impact of connectives on the memory for expository texts. Applied Cognitive Psychology 7, 317-339.

[31] Millis, K.K. \& Just, M. A. (1994). The influence of connectives on sentence comprehension. Journal of Memory and Language 26, 128-147.

[32] Murray, J.D. (1997). Connectives and narrative text: the role of continuity. Memory \& Cognition 25.2, 227-236.

[33] Nippold, M.A., Schwarz, I.E., Undlin, R.A. (1992). Use and understanding of adverbial conjuncts: a developmental study of adolescents and young adults. Journal of Speech and Hearing Research 35, 108-118.

[34] Olsen, L. \& Huckin, T.N. (1990). Point-driven understanding in engineering lecture comprehension. English for Specific Purposes 9, 33-47.

[35] Ozono, S. \& Ito, H. (2003). Logical connectives as catalysts for interactive L2 reading. System 31, 283-297.

[36] Pretorius, E.J. (2006). The comprehension of logical relations in expository texts by students who study through the medium of ESL. System 34, 432:450.

[37] Sanders, T.J.M. \& Noordman, L.G.M. (2000). The role of coherence relations and their linguistic markers in text processing. Discourse processes 29.1, 37-60.

[38] Sanders, T. J. M., Spooren, W. \& Noordman, L. (1993). Coherence relations in a cognitive theory of discourse representation. 
Cognitive Linguistics 4.2, 93-133.

[39] Swan, M. (1980). Practical English usage. Oxford: Oxford University Press.

[40] Taboada, M. (2006). Discourse markers as signals (or not) of rhetorical relations. Journal of Pragmatics 38, 567-592.

[41] Tasdemir, M. (2010). A comparison of multiple-choice tests and true-false tests used in evaluating student progress. Journal of Instructional Psychology 37.3, 258-266.

[42] van Dijk T.A. \& Kintsch, W. (1983). Strategies of discourse comprehension. New York: Academic Press

[43] Vekiri, I. (2002). What is the value of graphical display in learning? Educational Psychology Review 14.3, 261-312.

[44] Verdi, M.P. \& Kulhavy, R. (2002). Learning with maps and texts: An overview. Educational Psychology Review 14.1: 27-46.

[45] Vonk, W. \& Noordman, L.G.M. (1990). On the control of inference processes in text understanding. In D.A. Balota, G.F. Flores d'Arcais, \& K.Rayner (Eds.), Comprehension processes in reading (pp. 447-464). Hillsdale: NJ: Erlbaum.

Zorana Vasiljevic ( $\mathrm{PhD}$, University of Queensland) is associate professor at the Faculty of Language and Literature at Bunkyo University, Japan, where she teaches courses in English as a foreign language, L2 teaching methodology and materials development. Her research interests include L2 vocabulary acquisition, discourse analysis and EFL methodologies. 